\title{
Development of an Evidence-Based Clinical Practice Guideline of Korean Medicine for Stroke: A Study Protocol
}

\author{
Chang-ho Han ${ }^{1,3}$, Mikyung Kim ${ }^{2,3}$ \\ ${ }^{1}$ Department of Internal Medicine, College of Korean Medicine, Dongguk University \\ ${ }^{2}$ Department of Internal Medicine, College of Korean Medicine, Sangji University \\ ${ }^{3}$ Evidence Based Clinical Practice Guidelines Development Group (EBCPGsDG) for Stroke, \\ The Society of Stroke on Korean Medicine
}

\begin{abstract}
Introduction: The aim of this study protocol is to share and disclose the methodology used to develop an evidence-based clinical practice guideline (CPG) of therapeutic interventions used in Korean medicine for patients with stroke. Methods: The CPG development process will consist of two phases. In phase I, a development committee will be established, and they will decide the key questions to be answered. A systematic review and meta-analysis will be performed to answer these key questions by searching relevant randomized controlled trials and systematic reviews. Draft recommendations will be developed according to the evidence level and recommendation grades primarily determined using the GRADE methodology. Panels comprised of external experts will be formed, and surveys and a face-to-face meeting will be conducted to reach a consensus on the recommendations. A preliminary guideline will be created after final review by the development committee. In phase II, we will conduct clinical trials and economic analysis to supplement the lack of evidence found in the phase I.

Conclusion: The CPG is expected to help doctors practicing Korean medicine in clinics or hospitals with making decisions based on the most reliable evidence, ultimately leading to the provision of optimal care for patients with stroke.
\end{abstract}

Key Words : stroke, Korean medicine, clinical practice guideline, evidence-based medicine

\section{Introduction}

Stroke is one of the most important causes of death worldwide $^{1)}$ as well as in Korea ${ }^{2)}$. Although stroke mortality has been decreasing ${ }^{2}$, the absolute number of stroke patients is still considerable. The global burden of stroke is significant and continues to grow ${ }^{1)}$. Considering the trends in population aging, the incidence of stroke is expected to rise in the further ${ }^{2}$.

Although standardized stoke management guidelines have been published ${ }^{3,4)}$, stroke survivors receiving conventional treatment are still suffering from the sequelae and risk of recurrence of stroke. Patients with stroke are more likely to use complementary and alternative medicine, including traditional medicine (TM), than other populations ${ }^{5,6}$.

Korean medicine (KM), a branch of traditional East Asian medicine (TEAM) originated in ancient China and subsequently developed in the Korean peninsula. It comprises one of the two major axes of the medical

\footnotetext{
- Received : 14 March 2018

- Revised : 8 May 2018

- Accepted : 8 May 2018

- Correspondence to: Mikyung Kim

Department of Internal Medicine, College of Korean Medicine, Sangji University 83 Sangjidae-gil, Wonju,

Gangwondo, Republic of Korea

Tel : +82-33-741-9215, E-mail : mkim2017@sangji.ac.kr,01mkkim@gmail.com
} 
system alongside conventional medicine in Korea. The demand for $\mathrm{KM}$ is substantial, particularly among patients with stroke ${ }^{7}$. According to a recent survey conducted in Korea, stroke was the most common reason for admission to Korean medicine hospitals for the past one year ${ }^{7)}$.

A Clinical Practice Guideline (CPG) is a systematically developed statement to help medical staff and patients make appropriate decisions about the health care services provided in a particular clinical setting ${ }^{8}$.

A CPG is one of the most powerful and effective means of integrating evidence-based medicine into the clinical setting. It is not only a continuous and systematic way to improve the quality of medical care, but also provides high-quality information to patients ${ }^{8}$.

To keep up to date with worldwide efforts to implement evidence-based stroke care in clinical practice in Korea), guidelines for stroke management ${ }^{10)}$ and stroke rehabilitation ${ }^{11)}$ were published in 2009 and have been subsequently updated ${ }^{12,}{ }^{13)}$. However, these guidelines do not refer to TM used in Korea ${ }^{10,12)}$ and only briefly mention the effect of acupuncture on motor function ${ }^{11,13)}$. Given the considerable demand and use of $\mathrm{KM}$, it is necessary to develop evidence-based CPGs that include data from studies investigating KM.

In 2016, under the support of the Korean Ministry of Health and Welfare, a project commenced to develop CPGs for approximately 30 diseases or conditions including stroke ${ }^{14)}$. This study is part of this project. In 2008, a research team within the field of $\mathrm{KM}$ was established to develop CPGs for stroke. The team published a preliminary guideline of $\mathrm{KM}$ for stroke in 2010, summarizing the results of their review of current evidence on the effectiveness and safety of $\mathrm{KM}$ interventions ${ }^{15)}$.

Since then, studies on the clinical usefulness of TEAM for stroke have been actively conducted, and the new evidence is worth incorporating into CPGs.
Therefore, instead of amending the previous guideline, we have decided to prepare a new guideline using a more rigorous and transparent methodology, based on a comprehensive review of related literature such as randomized controlled trials (RCTs) and systematic reviews (SRs).

The aim of this study protocol is to share and disclose the methodology used to develop an evidence-based CPG of therapeutic interventions used in KM for patients with stroke.

\section{Methods/Design}

The KM standard CPG development project consists of two phases over five years. In phase I, within the first two years, we will determine key questions and obtain available literature to answer these questions. We will systematically review the documents that have been found, perform a meta-analysis that can be quantitatively synthesized, and develop a preliminary guideline based on the SR and meta-analysis of currently available evidence. In phase II, over the subsequent three years, we will focus on supplementing the lack of evidence found in the literature review by conducting clinical trials and an economic analysis, and then, update the preliminary guideline.

This protocol will mainly describe the methodology used during the first phase of the project in detail. To establish the methodology, we have referred to the preferred reporting items for the development of evidence-based CPGs in TM (PRIDE-CPG-TM) of Korea Institute of Oriental Medicine ${ }^{16)}$ and traditional Chinese medicine CPG development plans of China ${ }^{17,18)}$ and Hong Kong ${ }^{19)}$.

\section{Target Patients}

The target patients of this CPG are adults (18 years and older) with stroke. This CPG will focus on patients 
diagnosed with cerebrovascular disease or sequelae of wind stroke based on brain imaging or clinical diagnosis according to the Korean Standard Classification of Diseases (I60-69, or U23.4). The users of this guideline will be doctors of $\mathrm{KM}$ treating patients with stroke in clinics or hospitals. .

\section{Development Committee}

The CPG development committee will consist mainly of professors and residents who have majored in cerebrovascular diseases at colleges of KM. In addition, researchers who have graduated from the college of $\mathrm{KM}$, traditional Chinese medicine, or nursing will also be included.

\section{Key Questions}

'Are therapeutic interventions of KM helpful to improve specific outcomes in patients with stroke?' Within this core concept, the development committee will determine specific key questions through discussions. The range of therapeutic interventions covers acupuncture, electroacupuncture, moxibustion, herbal medicine, pharmacopuncture, and Tuina. The term stroke includes ischemic stroke, hemorrhagic stroke, and cases involving both, or cases where the stroke type is not specified.

\section{Literature review}

\subsection{Search Strategy}

Several core international databases such as PubMed, EMBASE, Cochrane Library, and China National Knowledge Infrastructure and domestic databases including Oriental Medicine Advanced Searching Integrated System and National Digital Science Library will be searched. The language will be restricted to English, Chinese, or Korean. The articles cited in the selected studies will also be reviewed. Dissertations as well as articles published in peer-reviewed journals will be included. Conference proceedings that do not fully provide the results of the original studies will be excluded. The search term will be determined according to the key questions. It will comprise of a combination of the outcomes and interventions from the key questions.

\subsection{Eligibility Criteria}

RCTs and SRs investigating the efficacy of interventions of KM in patients with stroke will be included. There will be no restrictions on the types or stages of stroke. Among the various outcomes, death, stroke recurrence, moderate to severe disability, and neurological deficit will be regarded as critical, and dysphagia, dysuria, shoulder pain, and quality of life will be considered important. Studies providing these critical or important outcomes measured using globally recognized assessment tools, or calculated in a standardized manner will be included.

The following are exclusion criteria: Studies that have evaluated the effects of combinations of various interventions, rather than a single intervention (e.g. acupuncture + moxibustion + herbal medicine), studies comparing interventions of which effects have not been sufficiently proven (e.g. reflexology, infrared irradiation), studies without any of the variables discussed above as critical or important outcomes, and studies that provide data that cannot be quantitatively synthesized.

\subsection{Quality Assessmen}

Risk of biases (RoBs) of the included RCTs will be assessed using the Cochrane RoB tools. The tool consists of seven domains including random sequence generation (selection bias), allocation concealment (selection bias), blinding of participants and personnel (performance bias), blinding of outcome assessment 
(detection bias), incomplete outcome data (attrition bias), selective reporting (reporting bias) and other bias $^{20)}$. RoBs of the selected SRs will be assessed using the Assessment of Multiple SysTemAtic Reviews (AMSTAR). AMSTAR consists of 11 domains including a priori design provision, duplicate study selection and data extraction process etc ${ }^{21)}$. Applying AMSTAR to the SRs published within last 10 years, SRs of higher quality (well documented four or more of the 11 domains of the AMSTAR) will be finally included $^{22)}$.

\subsection{Literature Selection}

Data Extraction, and Meta-analysis. More than two people per specific intervention will be assigned to review and select literature. Disagreements will be resolved by mediation with the corresponding author. Data will be extracted from the selected literature and meta-analysis will be performed using RevMan software.

\section{Evidence Level}

The evidence level of this CPG will be determined using the Grading of Recommendations, Assessment, Development and Evaluations (GRADE). According to the GRADE methodology, the evidence level is determined by considering the study design, RoB, inconsistency, indirectness, imprecision, and other considerations such as publication bias of the contained evidence $^{23)}$.

The study design for all included studies will be a RCT. The RoB will reflect the results from the application of the Cochrane RoB assessment to each article. Performance bias will not be considered when assessing the RoB in GRADE, because most of the interventions of $\mathrm{KM}$ are difficult to maintain patient and/or practitioner blinding. If any of the included studies that were evaluated as having a high RoB in the rest of the domains of the Cochrane RoB tool, the RoB in GRADE will be considered to be serious. Unclear RoB of the individual studies will not be considered to determine the evidence level using GRADE. Inconsistency will be determined based on the statistical heterogeneity between studies during the process of the meta-analysis. Inconsistency will be considered serious if the $\mathrm{I}^{2}$ value is greater than $75 \%{ }^{23,24)}$. Studies involving patients with ischemic, hemorrhagic, or nonspecific stroke will be assessed as not having serious indirectness. If the total number of subjects in a meta-analysis is less than 400 , or the $95 \%$ confidence interval of the meta-analysis overlaps the invalid interval, imprecision will be assessed as serious ${ }^{23,24)}$. In the case of including more than 10 studies in a meta-analysis, the possibility of publication bias will be checked by creating a funnel $\operatorname{plot}^{20,23)}$.

According to the GRADE methodology, the evidence level is categorized as high, moderate, low, or very low ${ }^{23}$. When publishing the final guideline, we will describe the evidence level that is classified as very low or that contains less than two articles for one key question as insufficient. The results of the meta-analysis performed using RevMan will be imported into the GRADEpro Guideline Development Tool ${ }^{25}$ and tabulated in the Summary of Finding Table $(\mathrm{SoF})^{25}$.

\section{Recommendation Grade}

This CPG will classify the recommendation level into four grades: A, B, C, and a Good Practice Point (GPP). Grade A recommends the use of the intervention, $\mathrm{B}$ indicates that the intervention should be considered, $\mathrm{C}$ indicates it may be considered for use, and the GPP indicates that the intervention is recommended based on the clinical experience of the CPG development committee. When drafting recommendations, the grades will be determined according to the evidence level 
derived by the GRADE as follows: A for high, B for moderate, $\mathrm{C}$ for low, and GPP for very low. All recommendations will go through the process of reaching a consensus, will be reviewed by the $\mathrm{CPG}$ development committee, and final recommendations and grades will be determined.

\section{Consensus Deduction}

Two panels will be formed and surveys and face-to-face meetings will occur to reach consensus on the recommendations. Panel 1 will be comprise representatives from nine major clinical subcommittees of the Society of KM including the society of stroke on KM. Panel 2 will consist of opening doctors, methodologists, humanities and social scientists, nursing management specialists, experts in health insurance review and assessment, civil society representatives and consumers.

Each panel will review the draft recommendations, and then two surveys will be performed to investigate their agreement with the recommendations and to receive feedback for revision. Recommendations that fail to reach consensus through the surveys will be discussed at a face-to-face meeting. Recommendations not reaching consensus through methods will not be included in the guideline.

\section{Reflection of Clinical Experiences}

We conducted an E-mail survey to investigate the current status of clinical practices of KM in stroke care $^{26}$. We plan to visit KM clinics and observe doctors in primary care treating patients with stroke. The findings of the survey and observations made will be reflected in the guideline. Through these efforts, we will make the CPG more practical, reflecting not only the outcomes from literature but also the experiences of clinical practitioners.

\section{Update Plan}

We will update the guideline every three years after publishing the first version. New scientific evidences identified through periodic monitoring of relevant literature will be incorporated into the updated guideline.

\section{Dissemination and Implementation}

To disseminate and implement the CPG, a summary and brief instructions for the CPG, and guidelines for patients will be developed. An implementation plan for the clinical setting will also be established. The CPG and its spreading tools will be posted through the following websites: Guideline Center for KM (http://www.g-kom.or.kr), KM Stroke Guideline Research Center (http://www.hanmed.kr), and The Society of Stroke on KM (http://kmstroke.or.kr).

\section{Discussion}

This study protocol describes the methodology that will be used to develop an evidence-based CPG of KM for stroke. Prior to the development of this guideline, evidence-based CPGs of TM have been or are being developed in East Asia.

In China, more than 500 CPGs have been published ${ }^{19}$. This includes five CPGs for stroke ${ }^{17-19)}$. Most of the CPGs were consensus-based rather than evidence-based guidelines, while the CPG published by the China Academy of Chinese Medical Sciences in 2011 adopted an evidence-based methodology ${ }^{18)}$. However, this guideline used its own evidence rating system developed by the Beijing university of Chinese medicine, rather than choosing more commonly used methodologies such as GRADE ${ }^{27,28)}$. Furthermore, its target population is patients soley with ischemic stroke ${ }^{27,28)}$. In 2015, Zhang et al. published a study evaluating the effects of acupuncture for stroke using the GRADE methodology ${ }^{24)}$. 
This study suggested acupuncture as a weak recommendation for to improve stroke rehabilitation including neurological deficits, dysphagia, and disability ${ }^{24)}$.

The Hong Kong Hospital Authority launched a project to develop evidence-based CPGs for three major diseases including ischemic stroke in $2013^{19)}$. The CPG for ischemic stroke is currently under development ${ }^{19)}$.

The CPG task force of the EBM Committee of the Japan Society for Oriental Medicine is disclosing a list of guidelines containing descriptions of traditional herbal remedies on their website ${ }^{29)}$. However, there is no guideline regarding TM for stroke at present ${ }^{29}$.

It is not easy to find evidence-based guidelines for stroke published worldwide that have referred to therapeutic interventions of TEAM including KM. According to a stroke guideline published by the Ottawa panel in Canada in 2006, acupuncture has limited effects for improving motor function and quality of life in patients with stroke ${ }^{30)}$. The Stroke engine in Canada describes acupuncture as having limited effects on improvement of motor function and activities of daily living (ADL). However, it is difficult to draw conclusions because most other areas of stroke have insufficient evidence ${ }^{31)}$. A CPG for stroke rehabilitation developed by the United States Veterans Affairs and Department of Defense suggested that there is a lack of evidence to recommend acupuncture for the purpose of improving stroke rehabilitation ${ }^{32)}$. According to another guideline published by the American Heart Association/American Stroke Association in 2016, acupuncture may be considered for patients with dysphagia ${ }^{3)}$. However, acupuncture is not recommended for improvement of ADL and upper limb function, and there is a lack of evidence on the effectiveness of acupuncture for shoulder pain, motor function, or walking mobility ${ }^{3)}$. The CPG for stroke management published by the Scottish Intercollegiate Guidelines
Network of the United Kingdom stated that there is a lack of evidence of the benefit of acupuncture for shoulder pain in stroke patients ${ }^{33)}$. In 2010, the National Institute for Health and Care Excellence (NICE) updated the evidence for stroke rehabilitation with the same content $^{34)}$. The NICE stroke guideline also derived recommendations based on SRs of not only acupuncture but other representative interventions of TEAM such as moxibustion and cupping. However, use of these interventions were not encouraged because of limited evidence $^{34)}$. The fourth edition of the CPG for stroke by the Royal College of Physicians stated that acupuncture should not be used for any purpose other than clinical trials ${ }^{35}$, and the next edition did not mention acupuncture or other related interventions at all ${ }^{36)}$. The stroke CPG published in New Zealand ${ }^{37)}$ and Australia ${ }^{38)}$ in 2010 did not recommend acupuncture. The 2017 revised version of the Australian guideline suggested that acupuncture can be recommended for post-stroke depression, however, did not recommend it for ADL, spasticity, or dysphagia ${ }^{39)}$. A Japanese CPG for stroke published in 2004 recommended use of electroacupuncture for shoulder problems ${ }^{40)}$. However, the updated guidelines that were published in 2009 no longer mentioned acupuncture ${ }^{41)}$.

In Korea, CPGs for stroke management ${ }^{10,12)}$ and rehabilitation $^{11,13)}$ have been published. The management guideline of the Korean Stroke Society did not mention any interventions of $\mathrm{TM}^{10,12)}$. The first version of the rehabilitation guideline did not recommend acupuncture for the purpose of improving motor function ${ }^{11)}$, and its revised version mentioned that there was a lack of evidence for the benefit of acupuncture ${ }^{13)}$.

In $\mathrm{KM}$ academia, a research team was established in 2008 to develop CPGs for and a preliminary guideline was published in $2010^{15}$. This is the result of a comprehensive review of the literature on the effect of $\mathrm{KM}$ for treatment of stroke. In addition, guidelines 
on the role of acupuncture for spasticity ${ }^{42)}$ and urinary retention $^{43)}$ in stroke patients have also been published.

The existing guidelines aforementioned for stroke have not been developed on the basis of rigorous evidence-based methodology such as GRADE, nor have they covered the use of a variety of interventions for different types of stroke. Furthermore, they do not adequately reflect the current status of KM practices performed for patients with stroke in Korea. Since the publication of the preliminary guideline of $\mathrm{KM}$ for stroke ${ }^{15)}$, numerous publications regarding clinical effectiveness of interventions of KM for stroke have been reported in recent years. Therefore, the current literature must be reviewed using rigorous methodology, which will help develop an updated CPG that reflects current practices in Korea.

\section{Conclusions}

The output of this project will be an evidence-based CPG of KM for stroke. The CPG is expected to help doctors of $\mathrm{KM}$ in clinics or hospitals to make informed decisions and provide optimal care for patients with stroke.

\section{Conflict of Interests}

The authors declare that they have no conflicts of interest.

\section{Authors' Contribution}

C-h Han conceptualized the project and designed the protocol. M Kim drafted the manuscript. C-h Han made critical revisions and completed the manuscript. M Kim had the final responsibility for the decision to submit this manuscript for publication. All authors read and approved the final manuscript.

\section{Acknowledgments}

This research was supported by a grant of the Korea Health Technology R\&D Project through the Korea Health Industry Development Institute (KHIDI), funded by the Ministry of Health \& Welfare, Republic of Korea (HB16C0001).

\section{References}

1. Feigin VL, Norrving B, Mensah GA. Global burden of stroke. Circulation research. 2017;120(3):439-48.

2. Hong KS, Bang OY, Kang DW, Yu KH, Bae HJ, Lee JS, et al. Stroke statistics in Korea: part I. Epidemiology and risk factors: a report from the korean stroke society and clinical research center for stroke. Journal of Stroke. 2013; 15(1):2-20.

3. Winstein CJ, Stein J, Arena R, Bates B, Cherney LR, Cramer SC, et al. Guidelines for adult stroke rehabilitation and recovery. Stroke. 2016;47(6): e98-e169.

4. Ahmed N, Steiner T, Caso V, Wahlgren N. Recommendations from the ESO-karolinska stroke update conference, Stockholm 13-15 November 2016. European Stroke Journal. 2017;2(2):95-102.

5. Liao CC, Lin JG, Tsai CC, Lane HL, Su TC, Wang HH, et al. An Investigation of the Use of Traditional Chinese Medicine in Stroke Patients in Taiwan. Evidence-Based Complementary and Alternative Medicine. 2012;2012:8.

6. Wells RE, Phillips RS, Schachter SC, McCarthy EP. Complementary and alternative medicine use among US adults with common neurological conditions. Journal of Neurology. 2010;257(11): 1822-31.

7. Ministry of Health and Welfare. The third 
utilization of oriental medical services survey 2014 (Korean). Available at:URL: http://www.ndsl.kr/ ndsl/commons/util/ndslOriginalView.do?cn= TRKO201600011606\&dbt=TRKO.

8. Darling G. The impact of clinical practice guidelines and clinical trials on treatment decisions. Surgical Oncology. 2002;11(4):255-62.

9. Goldstein LB, Bushnell CD, Adams RJ, Appel LJ, Braun LT, Chaturvedi S, et al. Guidelines for the primary prevention of stroke. A guideline for healthcare professionals from the American Heart Association/American Stroke Association. Stroke. 2010;42(2):517-84.

10. The writing group of clinical practice guideline for stroke. Clinical Practice Guideline for Stroke. 1st ed. Clinical Research Center for Stroke 2009.

11. Kim YH, Han TR, Jung HY, Chun MH, Lee J, Kim DY, et al. Clinical Practice Guideline for Stroke Rehabilitation in Korea. Brain \&Neurorehabilitation. 2009;2(1):1-38.

12. Hong KS, Yu KH, Rha JH, Kim HY, Koo J, Park $\mathrm{JM}$, et al. Review of Evidences and Updates of the Korean Clinical Practice Guidelines for Stroke in 2013. Journal of the Korean Neurological Association. 2013;31(3):143-57.

13. Rah UW, Kim YH, Ohn SH, Chun MH, Kim MW, Yoo WK, et al. Clinical Practice Guideline for Stroke Rehabilitation in Korea 2012. Brain \& Neurorehabilitation. 2014;7(Suppl 1):S1-S75.

14. Ministry of Health and Welfare. Launch of Korean Medicine Standard Clinical Practice Guideline Development Team (Korean). 2016; Available at:URL: http://www.mohw.go.kr/front_new/al/sal0301vw. jsp?PAR_MENU_ID $=04 \& M E N U \_I D=0403 \& p a$ $\mathrm{ge}=1 \&$ CONT_SEQ $=333629 \& \mathrm{SEARCHKEY}=\mathrm{TI}$ TLE\&SEARCHVALUE $=\% \mathrm{C} 7 \% \mathrm{~A} 5 \% \mathrm{C} 1 \% \mathrm{D} 8 \%$ C0\%D3\%BB\%F3\%C1\%F8\%B7\%E1\%C1\%F6 $\% \mathrm{C} 4 \% \mathrm{~A} 7$.

15. Han CH. Clinical Practice Guideline of Korean
Medicine for Stroke : Preliminary Guideline and Recommendation. The Journal of Internal Korean Medicine. 2012;33(4):347-66.

16. Choi J, Choi TY, Jun JH, Lee JA, Lee MS. Preferred reporting items for the development of evidencebased clinical practice guidelines in traditional medicine (PRIDE-CPG-TM): explanation and elaboration. European Journal of Integrative Medicine. 2016;8(6):905-15.

17. Chen ZG, Luo H, Xu S, Yang Y, Wang SC. Study on the methodology of developing evidence-based clinical practice guidelines of chinese medicine. Chinese journal of integrative medicine. 2015;21(11):874-80.

18. Ya Y, Shi NN, Han XJ, Gao Y, Xu JL, Liu DS, et al. Appraisal of clinical practice guidelines for ischemic stroke management in Chinese medicine with appraisal of guidelines for research and evaluation instrument: A systematic review. Chinese journal of integrative medicine. 2015;21(9):707-15.

19. Shi N, Zhong LL, Han X, Ziea TC, Ng B, Bian $Z$, et al. Enhanced evidence-based Chinese medicine clinical practice guidelines in Hong Kong: a study protocol for three common diseases. Evidence-Based Complementary and Alternative Medicine. 2015;2015.

20. Higgins JP, Green S. Cochrane handbook for systematic reviews of interventions. Vol. 4. John Wiley \& Sons. 2011.

21. Shea BJ, Hamel C, Wells GA, Bouter LM, Kristjansson E, Grimshaw J, et al. AMSTAR is a reliable and valid measurement tool to assess the methodological quality of systematic reviews. Journal of clinical epidemiology. 2009;62(10):1013-20.

22. Canadian Agency for Drugs and Technologies in Health (CADTH). Intervention Directed to Consumers. Available at:URL: https://www.cadth.ca/ interventions-directed-consumers.

23. Schünemann H, Brożek J, Guyatt G, Oxman A. 
GRADE handbook for grading quality of evidence and strength of recommendations. Updated October 2013. The GRADE Working Group. 2013. 2014.

24. Zhang X, Liu XT, Kang DY. GRADE in systematic reviews of acupuncture for stroke rehabilitation: recommendations based on high-quality evidence. Sci Rep. 2015;5:16582.

25. GRADE Working Group. GRADEpro GDT. Available at:URL: https://gradepro.org/.

26. Kim MK, Han CH. E-mail survey on the current status of clinical practice and activation measures for Korean medicine in stroke care. The Journal of Korean Medicine. 2017;38(3):143-59.

27. Zhou L, Chen Y, Liu J, Liu Zl, Gao Y. Evaluating the implementation of evidence-based TCM Clinical Practice Guidelines for Cerebral Infarction. European Journal of Integrative Medicine. 2014;6(2):147-55.

28. China Academy of Chinese Medical Sciences. Evidence-based guidelines of clinical practice in Chinese medicine. 2011.

29. Japan Society for Oriental Medicine EBM Committee. Clinical Practice Guidelines Containing Kampo Products in Japan. Available at:URL: http://www.jsom.or.jp/medical/ebm/.

30. Ottawa Panel. Ottawa panel evidence-based clinical practice guidelines for post-stroke rehabilitation. Topics in stroke rehabilitation. 2006;13(2):1-269.

31. Canadian Partnership for Stroke Recovery. Stroke Engine. Available at:URL: https://www.strokengine. ca/clinician-info/acupuncture-clinician-info/.

32. Management of Stroke Rehabilitation Working Group. VA/DOD Clinical practice guideline for the management of stroke rehabilitation. Journal of rehabilitation research and development. 2010;47(9):1-43.

33. Smith L. Management of Patients With Stroke:
Rehabilitation, Prevention and Management of Complications, and Discharge Planning: a National Clinical Guideline. SIGN. 2010.

34. NICE. 2010 Evidence Update on Stroke Rehabilitation. Available at:URL:https://arms. evidence.nhs.uk/resources/hub/37918/attachment

35. Intercollegiate Stroke Working Party. National clinical guideline for stroke. 4th ed. Royal College of Physicians. 2012.

36. Intercollegiate Stroke Working Party. National clinical guideline for stroke. 5th ed. Royal College of Physicians. 2016.

37. Stroke Foundation of New Zealand and New Zealand Guidelines Group. Clinical Guidelines for Stroke Management 2010. Wellington: Stroke Foundation of New Zealand; 2010.; Available at:URL: http://www.stroke.org.nz/resources/NZ ClinicalGuidelinesStrokeManagement2010Activ eContents.pdf.

38. Boddice G, Brauer S, Gustafsson L, Kenardy J, Hoffmann T. Clinical Guidelines for Stroke Management 2010. Melbourne, Australia: National Stroke Foundation. 2010.

39. National Stroke Foundation. Clinical Guidelines for Stroke Management 2017 (draft). Melbourne Australia.; Available at:URL: http://www.opalinstitute. org/uploads/1/5/3/9/15399992/draft_clinical_guidelines_ for_stroke_management_2017_summary_of_re commendations_public_consultation_1_.pdf.

40. Chuma T. Stroke Treatment Guideline. The Japanese Journal of Rehabilitation Medicine. 2011;48(2):117-20.

41. Shinohara Y. Japanese Guidelines for the Management of Stroke 2009 : important revised points necessary for the neurologist. Rinsho shinkeigaku = Clinical Neurology. 2010;50(11): 808-11.

42. Kim JS, Shin SW, Lee1 EJ, Shin BC, Lee MS, 
Lim SM, et al. Clinical Practice Guideline on Acupuncture for Post-stroke Spasticity. Journal of Korean Medicine. 2015;36(1):1-8.

43. Lee JW, Lee EJ, Shin BC, Lee MS, Lim SM, Cho CS, et al. Clinical Practice Guideline for acupuncture in Post-stroke urinary retention. Journal of Korean Medicine. 2016;37(1):1-9.

\section{ORCID}

Chang-ho Han: https://orcid.org/0000-0003-3460-9263

Mikyung Kim: https://orcid.org/0000-0002-9912-6249 